\title{
Les États-Unis et « l'autre crise » : l'aide extérieure américaine à l'épreuve de la crise alimentaire mondiale
}

The United States and the "other crisis ": US foreign aid and the challenge of the food crisis

Los Estados Unidos y la « otra crisis " : la ayuda exterior estadounidense a prueba de la crisis de alimentos

Jean-Baptiste Velut

\section{OpenEdition}

\section{Journals}

Édition électronique

URL : https://journals.openedition.org/ideas/585

DOI : 10.4000/ideas.585

ISSN : 1950-5701

Éditeur

Institut des Amériques

\section{Référence électronique}

Jean-Baptiste Velut, « Les États-Unis et « l'autre crise » : l'aide extérieure américaine à l'épreuve de la crise alimentaire mondiale », IdeAs [En ligne], 4 | 2013, mis en ligne le 15 octobre 2013, consulté le 20 octobre 2022. URL : http://journals.openedition.org/ideas/585 ; DOI : https://doi.org/10.4000/ideas. 585

Ce document a été généré automatiquement le 20 octobre 2022

\section{(c) (i) (2) $\Theta$}

Creative Commons - Attribution - Pas d'Utilisation Commerciale - Pas de Modification 4.0 International - CC BY-NC-ND 4.0

https://creativecommons.org/licenses/by-nc-nd/4.0/ 


\title{
Les États-Unis et « l'autre crise »: l'aide extérieure américaine à l'épreuve de la crise alimentaire mondiale
}

\author{
The United States and the " other crisis »: US foreign aid and the challenge of \\ the food crisis \\ Los Estados Unidos y la « otra crisis » : la ayuda exterior estadounidense a \\ prueba de la crisis de alimentos
}

Jean-Baptiste Velut

\section{« L'autre crise »}

1 Entre 2007 et 2010, la crise financière et ses conséquences économiques et sociales ont dominé l'actualité internationale et monopolisé l'attention des représentants du nouvel ordre mondial dont le G20 est l'un des principaux avatars. Dans l'ombre de cette tempête économique mondiale, «l'autre crise », c'est-à-dire la hausse vertigineuse des prix des aliments et ses conséquences humaines désastreuses pour les populations $d u$ Sud, n'a pas suscité une mobilisation politique comparable. Sans rester impassible à la crise alimentaire, les chefs d'État du G20 se sont concentrés sur les conséquences intérieures de la tempête économique et financière, reléguant l'aide publique au développement (APD) au second rang de leurs priorités. Mais si la flambée des prix des aliments a précédé de quelques mois la crise financière mondiale, cette dernière a indéniablement exacerbé les effets de la crise alimentaire. En tant qu'épicentre de ce séisme économique mondial - et en vertu de certaines décisions politiques qui seront évoquées ultérieurement -, les États-Unis ont une part de responsabilité au moins indirecte dans "l'autre crise ». En outre, si l'on peut contester l'idée d'obligation morale ou de responsabilité humanitaire, il est difficile de nier les risques d'instabilité 
politique et les enjeux sécuritaires associés à la crise alimentaire. D'un point de vue géopolitique, les États-Unis auraient donc tout intérêt à endiguer ce que l'Organisation des nations unies pour l'alimentation et l'agriculture (FAO) décrit comme un « tsunami silencieux ». L'objectif du présent article consiste à analyser les réponses que les ÉtatsUnis ont tenté d'apporter à la crise alimentaire. L'étude des mesures provisoires américaines s'accompagne d'une réflexion sur les récentes évolutions de l'APD aux États-Unis, tant du point de vue de ses objectifs que de ses modalités et de ses lacunes.

2 Avant d'étudier les réponses politiques du gouvernement américain face à la crise alimentaire mondiale, il convient de préciser les dimensions conjoncturelles et structurelles de ce phénomène. Le concept de "crise alimentaire » fait référence à l'envolée des prix des denrées alimentaires sur les marchés mondiaux qui a débuté à la fin de l'année 2006. L'économie mondiale a été frappée par deux vagues inflationnistes : une première période, entre la fin de l'année 2006 et le milieu de l'année 2008, au cours de laquelle l'indice des prix réels des aliments a augmenté de près de $80 \%$; un second cycle inflationniste dans la deuxième moitié de l'année 2010, au cours duquel les prix alimentaires ont connu une hausse de $40 \%$ avant de se stabiliser. Au total, entre septembre 2006 et juin 2011, les denrées alimentaires ont subi une inflation de $87 \%$. Cette flambée des prix n'a épargné aucun produit : le cours des céréales a augmenté de $110 \%$, les produits laitiers de $88 \%$, les huiles de $127 \%$, et la viande de $47 \%$ (voir Figure $1)^{1}$.

FIGURE 1 :

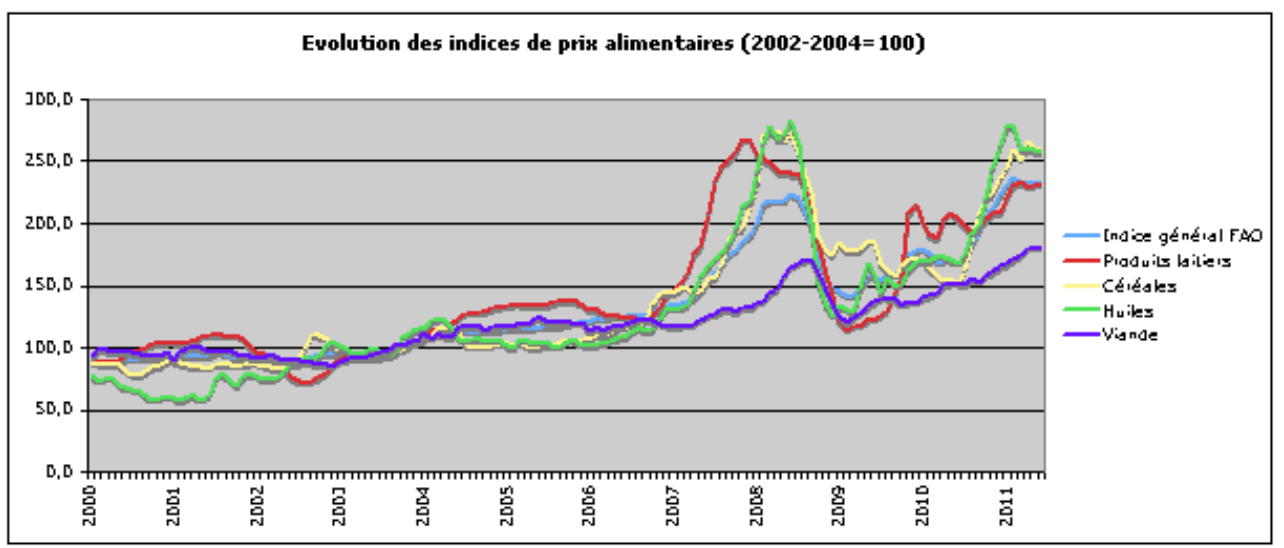

Source : FAO.

3 Cette inflation spectaculaire a eu des conséquences humanitaires dramatiques. Le nombre de personnes sous-alimentées est passé de 873 millions sur la période 2004-2006 à plus d'un milliard en 2009, un record historique depuis 1970 (FAO, 2009).

4 L'ampleur de cette catastrophe humanitaire va de pair avec son envergure géographique sans précédent. La crise a en effet frappé près de soixante pays, donnant lieu à une multitude d'émeutes aux quatre coins du monde, de l'Égypte à l'Indonésie en passant par Haïti et le Cameroun ${ }^{2}$. Les populations sous-alimentées se concentrent principalement en Asie et dans l'Afrique subsaharienne.

5 La flambée des prix des aliments s'explique par une série de facteurs conjoncturels et structurels. Premièrement, elle est le résultat de mauvaises récoltes dues à des intempéries régionales en Australie, en Europe de l'Est et au Canada (CRS, 2008: 2). La 
plupart des analystes ont néanmoins tendance à minimiser l'impact de ces facteurs à court terme, en soulignant notamment la hausse globale de la production céréalière mondiale entre 2006 et 2008 (FAO, 2009; Sen, 2008) ${ }^{3}$. Deuxièmement, la crise alimentaire est étroitement liée à l'envolée du cours du pétrole. Celle-ci s'est directement répercutée sur les coûts de production dans la mesure où le prix des engrais a presque triplé et les coûts de transport ont doublé sur la période 2006-2008 (FAO, 2008). Troisièmement, la production d'agrocarburants (à base de graines de céréales, d'oléagineux, de canne ou betterave à sucre), devenue plus rentable du fait de l'inflation du baril de Brent, a exacerbé ces pressions inflationnistes 4 . Les politiques de soutien à la production de ces nouveaux « biocarburants » aux États-Unis et en Europe ont par ailleurs contribué à réduire la surface cultivable consacrée à la production alimentaire et ainsi accentué la hausse des prix ${ }^{5}$. Quatrièmement, les restrictions des exportations adoptées par de nombreux pays en réponse à la crise ont limité l'offre alimentaire sur le marché mondial. Enfin, la spéculation sur les marchés à terme agricoles aurait aggravé ces effets (Rose, 2009).

6 L'inflation des denrées alimentaires est néanmoins bien plus qu'une simple évolution conjoncturelle et reflète de profondes mutations de l'économie mondiale. Elle est d'une part liée aux besoins énergétiques croissants des économies émergentes et à leur effet inflationniste; et, d'autre part, à l'évolution des habitudes alimentaires des nouvelles classes moyennes. L'amélioration du niveau de vie dans les pays émergents s'est traduite à la fois par une croissance de la demande alimentaire mondiale et par une hausse de la consommation en viande, dont la production mondiale requiert de plus en plus de matières végétales ${ }^{6}$. Cette nouvelle demande a de surcroît contribué à alimenter l'inflation des denrées alimentaires qui, comme le souligne l'économiste Amartya Sen, n'a pas épargné les populations pauvres au sein même des pays émergents (Sen, 2008). Les effets de la crise alimentaire ont été aggravés par la crise financière mondiale. Cette dernière a eu des effets dévastateurs sur les économies vulnérables, privées des remèdes qui les avaient auparavant aidées à lutter contre la pauvreté et la sousalimentation. Non seulement les investissements étrangers directs vers les pays en voie de développement ont chuté, mais les transferts de fonds des populations immigrées (remittances) et les exportations ont accusé un net repli (FAO, 2009).

7 Les États-Unis restent le premier pourvoyeur d'aide alimentaire au monde: leur contribution représente près de la moitié des ressources du Programme alimentaire mondial des nations unies (PAM) (48,5\% sur la période 1998-2008) et joue donc un rôle prépondérant dans l'assistance de millions de personnes sous-alimentées dans le monde (World Food Programme, 2009). Il apparait donc important d'analyser les mesures que le gouvernement américain a adoptées face à cette crise humanitaire sans précédent. Les États-Unis se sont-ils contentés de mesures à court terme ou ont-ils cherché à s'attaquer aux racines profondes de la crise? Quels atouts et/ou quelles faiblesses de la politique de l'aide extérieure américaine "l'autre crise» a-t-elle révélés?

\section{L'administration Bush à l'épreuve de la crise alimentaire}

8 L'administration de George W. Bush a marqué l'histoire des relations internationales par sa politique militaire interventionniste et son unilatéralisme. Pourtant, en dehors 
des questions sécuritaires, le quarante-troisième président a mené une politique économique internationale tout aussi offensive, tant au niveau de ses initiatives commerciales ${ }^{7}$ que de sa politique de l'aide extérieure. Parent pauvre de l'après-guerre froide, l'APD est redevenue un pilier stratégique de la politique étrangère américaine au lendemain des attentats du 11 Septembre. La stratégie de sécurité nationale (National Security Strategy) de 2002 souligne la nécessité de réformer la politique d'APD dans le but de promouvoir le développement économique et de construire « l'infrastructure de la démocratie » essentielle à la lutte contre le terrorisme selon George W. Bush (Bush, 2002: section VII). Ce dernier a indéniablement transformé le processus de l'aide extérieure et ce, à trois principaux égards : 1) en augmentant considérablement le budget de l'aide extérieure ${ }^{8}$ - de 12,6 milliards de dollars en 2000 à 26,3 milliards en $2008^{9}$; 2) en lançant de nouvelles initiatives politiques parmi lesquelles le Millennium Challenge Account (MCA $)^{10}$ et le Plan d'urgence du Président pour la lutte contre le Sida (PEPFAR) ; 3) en tentant de rationaliser le processus de décision de l'aide extérieure. Selon Henrietta Fore, directrice d'USAID de 2007 à 2008 : « L'administration Bush a sans aucun doute élevé l'image de [l'aide au] développement comme aucun président ne l'avait fait depuis Truman » (cité dans LaFranchi, $2008: 2$ ).

9 Cette déclaration est contestable si l'on considère l'héritage de John F. Kennedy, père de l'Agence pour le développement international (USAID) et architecte des programmes Peace Corps et Alliance for Progress. En outre, l'idée selon laquelle George W. Bush aurait transformé la politique de l'aide extérieure américaine doit toutefois être nuancée. D'une part, l'augmentation du budget de l'APD américaine est beaucoup plus modeste si l'on exclut les deux premiers bénéficiaires que sont l'Irak et l'Afghanistan ${ }^{11}$. D'autre part, la croissance remarquable du montant de l'aide extérieure sous la présidence Bush occulte son niveau historique relativement bas par rapport au total des dépenses fédérales américaines. Estimé à $1,2 \%$ du budget 2008, ce ratio est selon le Congressional Research Service inférieur à son niveau moyen pendant les années $1990(1,4 \%)$ et les années 1980 (1,8 \%) (Epstein, 2009 : 15). Enfin, les réformes de George W. Bush dans le domaine de l'aide extérieure se sont traduites par un affaiblissement de l'USAID, organe central de l'APD et de l'aide alimentaire, au profit de la Millennium Challenge Corporation et du département de la Défense ${ }^{12}$. Néanmoins, si l'on peut désapprouver la nouvelle orientation de l'aide extérieure sous l'administration Bush - au motif de son instrumentalisation à des fins militaires ou de la fragmentation du processus décisionnel $1^{13}$ - il est plus difficile d'ignorer le nouvel élan que le gouvernement républicain a donné à cet instrument de la politique étrangère américaine.

Lors de la crise alimentaire, les dirigeants américains tardent quelque peu à réagir, à l'instar de leurs homologues européens. Ce n'est en effet qu'au printemps 2008 que le président Bush demande au Congrès de puiser dans les ressources du Bill Emerson Humanitarian Trust - un fonds de réserves céréalières et financières géré par le département de l'Agriculture. Il parvient à obtenir une aide alimentaire d'urgence de 200 millions de dollars. Au total, le montant de l'aide alimentaire américaine augmente de $27 \%$ en 2008, portant le montant de l'aide américaine à près de 2,6 milliards de dollars, un niveau record au cours de la dernière décennie (voir figure 2) ${ }^{14}$. Malgré cette hausse notable (voir figure 2), la contribution des États-Unis au Programme alimentaire mondial recule légèrement (de $44 \%$ à $41 \%$ entre 2007 et 2008), en raison de la forte mobilisation de la communauté internationale - le budget du PAM ayant presque doublé entre 2007 et 2008 (WFP, 2009). 
11 En mai 2008, George W. Bush sollicite de nouveau le pouvoir législatif pour débloquer des crédits supplémentaires d'un montant de 770 millions de dollars pour l'année fiscale 2009, dans le cadre de la President's Food Security Response Initiative (PFSRI Intervention du Président pour la sécurité alimentaire). Imputée sur les crédits alloués pour le financement des guerres d'Irak et d'Afghanistan (Hamrahan, 2008), cette aide comprend deux volets: 590 millions de dollars pour l'acheminement de denrées alimentaires en urgence, et 180 millions pour l'aide au développement agricole par le financement de projets visant à réduire l'insécurité alimentaire à travers des projets à long terme (nutrition, approvisionnement en eau, hygiène, etc.) (USAID, 2009). Distribuées par l'intermédiaire du PAM et d'ONG internationales (Mercy Corps, Catholic Relief Services, etc.), ces denrées alimentaires sont destinées avant tout à l'Afrique et, dans une moindre mesure, à l'Asie centrale.

12 Si ces efforts traduisent une réelle volonté d'assister les pays en difficulté, ces chiffres méritent d'être interprétés avec précaution. L'augmentation à court terme des crédits alimentaires masque un phénomène structurel de fragilisation du processus de l'aide alimentaire déjà en cours avant la crise. Cette tendance se caractérise par une diminution des crédits annuels fixes (annual appropriations), au profit de l'aide allouée en urgence via des crédits additionnels (supplemental appropriations) (US GAO, 2007). Enfin, il serait vain d'évaluer le montant de l'aide alimentaire américaine sans le comparer aux autres dépenses du budget américain. Même à son point culminant en 2008, la somme allouée par les États-Unis à la crise alimentaire ne représente que $0,09 \%$ des dépenses du gouvernement fédéral, un montant inférieur au coût hebdomadaire de la guerre en Irak en 2008 (estimé à environ 2,8 milliards par semaine) $)^{15}$.

FIGURE 2 :

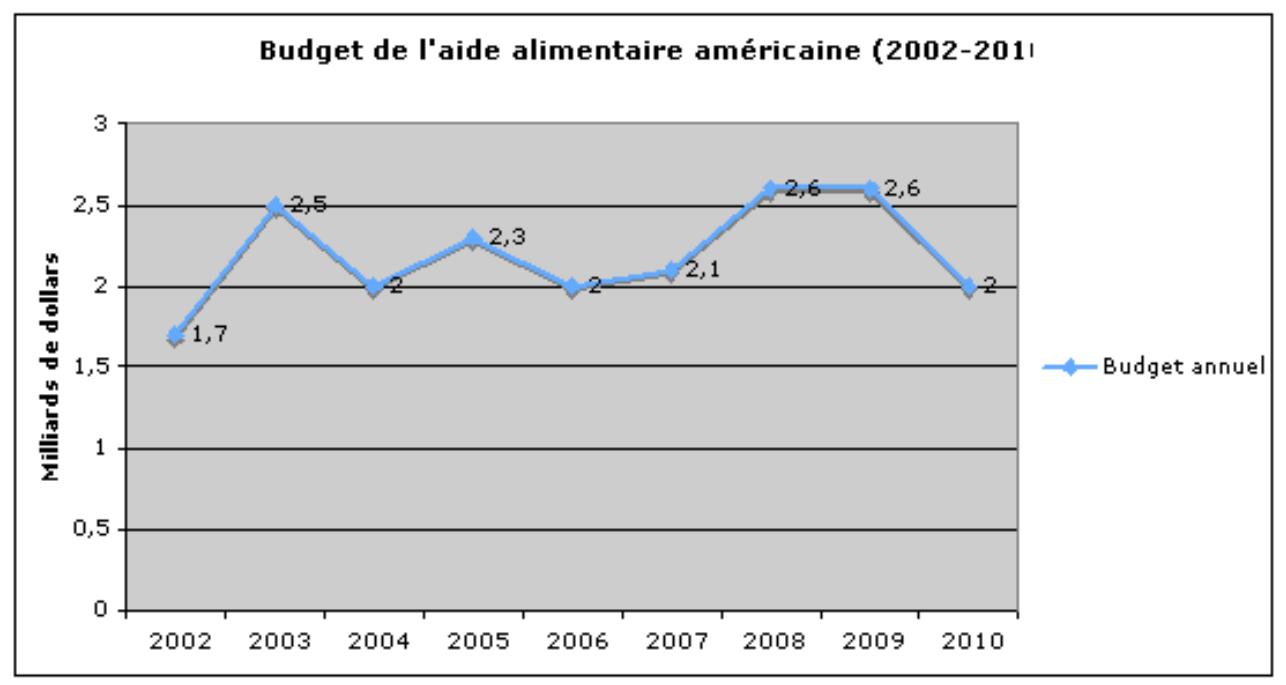

Sources : USAID, USDA, State Department

\section{Les déficiences structurelles de l'aide alimentaire}

Qu'on la juge parcimonieuse ou généreuse, l'aide extérieure américaine n'a jamais été entièrement désintéressée, dans la mesure où elle soutient à la fois l'agriculture et 
l'industrie du transport des États-Unis. Le Food for Peace Act de 1954 prévoit en effet que la majorité de l'aide alimentaire soit non seulement issue de la production agricole des États-Unis, mais également convoyée par des transporteurs maritimes américains ${ }^{16}$. Ces contraintes logistiques réduisent l'efficacité des efforts humanitaires de Washington à plus d'un titre. Premièrement, elles alourdissent le coût des denrées alimentaires. Une récente étude du Government Accountability Office (GAO) a démontré que l'utilisation de produits régionaux ou locaux réduirait le coût de l'aide américaine de $34 \%$ en Afrique subsaharienne et de $29 \%$ en Asie (US GAO, 2009). Par ailleurs, les transferts directs des récoltes céréalières américaines ont parfois des effets pervers sur les économies que les Etats-Unis sont censés secourir dans la mesure où ils tendent à déprécier les produits locaux ou régionaux. Ils risquent donc d'appauvrir les agriculteurs et de dissuader les investissements dans l'agriculture locale. Cette pratique de longue date, que certains apparentent à une forme de dumping, a fait récemment l'objet de vives critiques aussi bien de la part des associations humanitaires que des défenseurs du libre marché (Oxfam, 2005). Au milieu de la crise, ces débats ont pris une nouvelle ampleur lorsque l'ONG américaine CARE a officiellement annoncé qu'elle n'accepterait plus de marchandises agricoles américaines dans le cadre de ses opérations humanitaires. George W. Bush a lui-même désavoué cette pratique au cours de sa présidence en demandant à maintes reprises au Congrès de réduire la part des transferts directs au profit des achats locaux ou triangulaires (produits par un pays tiers). En 2007, l'administration républicaine a tenté de faire passer la part de l'aide alimentaire allouée aux produits locaux et régionaux à $25 \%$ dans le cadre du projet de loi agricole de 2008 (USDA, 2007). Le Congrès s'y est opposé en rappelant les enjeux intérieurs qui soustendent la politique de l'aide extérieure américaine :

Les Représentants [...] mettent en garde le pouvoir exécutif contre des propositions qui menacent l'équilibre subtil d'une coalition d'intérêts qui sert la cause des programmes d'aide alimentaire internationaux depuis plus de 50 ans (cité dans Hamrahan, 2008 : 11).

14 La loi agricole de 2008 a finalement créé un projet pilote autorisant l'achat de produits locaux et régionaux à hauteur de 60 millions de dollars sur cinq ans, une goutte d'eau dans le budget de l'aide alimentaire américaine. Depuis la décision du Canada de renoncer aux transferts directs en 2008, les États-Unis sont l'un des derniers pays industrialisés dont la majorité de l'aide alimentaire est conditionnée à l'achat de produits nationaux ${ }^{17}$.

15 Deuxièmement, le surcoût de transport lié à la situation quasi-monopolistique des convoyeurs américains - censés assurer au moins $75 \%$ du transport des marchandises réduit considérablement l'efficacité de l'aide extérieure. En 2007, une analyse du GAO a révélé que $65 \%$ de l'aide alimentaire était liée aux frais de transport. Sur la période 2001-2006, la hausse des dépenses logistiques - due en partie à la multiplication des situations d'urgence alimentaire - aurait contribué à un déclin de $52 \%$ du tonnage des marchandises livrées. Au vu de l'augmentation des coûts énergétiques, il est fort probable que cette perte de "productivité » de l'aide alimentaire se soit prolongée après 2006. En dehors de son surcoût, le système de préférence nationale de l'aide alimentaire américaine occasionne également d'importants délais dans l'acheminement des denrées alimentaires. Entre quatre et six mois séparent la commande de l'aide alimentaire de sa livraison dans la région en crise, une livraison qui arrive parfois après la période de pénurie voire en même temps que la nouvelle récolte locale - au risque de déstabiliser les marchés locaux. Depuis 2004-2005, certaines mesures adoptées après le 
tsunami asiatique ont permis de réduire les délais de l'aide alimentaire d'urgence. Toutefois, sans une véritable réforme du Food for Peace Act, le processus de l'aide alimentaire américaine, quelque populaire qu'il soit parmi les défenseurs des intérêts agricoles américains, restera lent, coûteux et parfois même contre-productif (US GAO, 2007).

16 Le troisième problème structurel de l'aide alimentaire américaine réside dans son manque de coordination. Cette déficience est dans une plus large mesure le fait de l'éclatement $\mathrm{du}$ processus décisionnel de l'aide extérieure, auquel participent non moins de cinquante agences gouvernementales (Brainard, 2008). Bien qu'ayant aggravé ce problème de fragmentation par la création de nouvelles institutions lors de son premier mandat, le président Bush s'est par la suite efforcé de rationaliser la politique de l'aide extérieure à travers une série d'initiatives comme la création du poste de Directeur de l'aide extérieure (chargé de coordonner l'APD) et l'intégration de certains programmes d'assistance gérés par l'Agence pour le développement international et le département d'État (Radelet, 2009). Parallèlement, la nouvelle Directrice de l'aide extérieure, Henrietta Fore, a créé la USAID Food Security and Food Price Increase Task Force (Groupe de travail sur la sécurité alimentaire et l'inflation alimentaire) dans l'espoir de coordonner les efforts de l'Agence pour le développement et de ses partenaires institutionnels (USAID \& USDA, 2008). Bien qu'incomplètes ${ }^{18}$, ces mesures représentent un premier pas vers une rationalisation du processus décisionnel de l'aide extérieure, notamment alimentaire.

17 Toutefois, améliorer la cohérence de la politique de l'aide alimentaire ne suffira pas si d'autres politiques vont à l'encontre de ses objectifs. Malgré ses tentatives de réformer l'aide extérieure, George W. Bush a défendu tout au long de sa présidence une politique énergétique qui a exacerbé les effets de la crise alimentaire mondiale. En pleine période d'inflation galopante, le Congrès et le Président ont soutenu l'expansion des surfaces cultivables destinées à la production d'agrocarburants, pourtant largement responsables de la flambée des prix des denrées alimentaires - avant d'acheminer l'aide humanitaire vers les pays en crise ${ }^{19}$. Ces politiques contradictoires ne sont toutefois pas l'apanage des États-Unis: l'Union européenne (premier producteur de biodiesel mondial), l'Inde, le Brésil et la Chine, tous pourvoyeurs d'aide humanitaire auprès du PAM ont pris des mesures pour accroître leur production de biocarburants (CNUCED, 2008). Aux États-Unis, ce problème est non seulement lié à la faible importance accordée à la politique d'aide extérieure, mais également à l'absence de vision stratégique du développement à long terme, une lacune de la politique étrangère qui se manifeste aussi par le refus de l'administration Bush de s'engager dans la lutte contre le réchauffement climatique - une autre menace à long terme pour la sécurité alimentaire des pays les plus pauvres.

\section{Vers une nouvelle politique de l'aide alimentaire?}

18 En somme, la politique de l'aide extérieure américaine, bien qu'indispensable à la gestion de la crise alimentaire mondiale, continue à souffrir de déficiences structurelles qui limitent considérablement son efficacité : un éclatement institutionnel, un système de préférence nationale lourd et coûteux et un manque de vision à long terme. Quelle réponse Barack Obama a-t-il apportée à ces défaillances? De prime abord, le Président démocrate semble tout aussi déterminé que son prédécesseur à faire de l'aide 
extérieure un pilier de sa politique étrangère. L'administration Obama a souligné l'importance du "smart power", qui consisterait, selon la secrétaire d'État Hillary Clinton, à mobiliser "l'ensemble des outils à notre disposition - diplomatiques, économiques, militaires, politiques, légaux et culturels - choisir le bon outil, ou la bonne combinaison d'outils en fonction de la situation " (Clinton, 2009). En dehors de sa dimension rhétorique, ce concept témoigne de la réelle volonté du Président démocrate de rééquilibrer une politique étrangère qu'il juge trop militariste. Cette volonté se traduit par des mesures concrètes comme le Bilan quadriennal de la diplomatie et du développement (Quadriennal Diplomacy and Development Review) du département d'État, qui associe ce dernier à l'Agence pour le développement international et à la Millennium Challenge Corporation afin de réévaluer les grands défis internationaux et d'optimiser les stratégies diplomatique et développementale américaines (US DOS, 2009b). Bien que le QDDR vise à apporter plus de cohérence à la politique de l'aide extérieure à travers une "approche gouvernementale globale » (whole of government approach), les détails de cette coordination entre les services américains restent flous, comme le souligne une récente étude du Congressional Research Service (CRS, $2011:$ 19-20).

19 Depuis sa campagne électorale, Barack Obama a affirmé à maintes reprises son ambition de doubler le montant de l'aide extérieure américaine (Obama, 2007 ; US DOS, 2009), une promesse qui s'est révélée irréalisable dans le climat actuel d'austérité. Malgré une nette augmentation du budget des affaires étrangères entre 2008 et 2010 (de 42,9 milliards de dollars en 2008 à 52,6 en 2009 et 55 en 2010), le Département d'État figure parmi les premières victimes du compromis budgétaire négocié entre la Maison Blanche et la majorité Républicaine en avril 2011, et devrait perdre 8,5 milliards de dollars en 2011, soit 15 \% du budget de 2010 (DOS, 2009c, 2011; Washington Post, 2011). Dans le domaine de l'aide alimentaire, la tendance est, à première vue, au déclin : la part du budget pour l'année 2010 ( 2 milliards de dollars) et 2011 ( 2 milliards) se situe bien en dessous du niveau de 2009 (2,6 milliards), malgré les fortes pressions inflationnistes subies par les denrées alimentaires au cours de la deuxième moitié de l'année 2010 (voir figure 2) 2 .

20 Cette tendance à la baisse des budgets alimentaires occulte toutefois la nouvelle impulsion que l'administration a donnée à l'aide extérieure. Lors du sommet du G8 de L'Aquila en juin 2009, l'administration américaine a promis d'investir 3,5 milliards de dollars sur trois ans dans l'agriculture des pays les plus pauvres, jouant ainsi un rôle clé dans la mobilisation de fonds publics et privés (comme la Bill \& Melinda Gates Foundation) contre l'insécurité alimentaire. En mai 2010, la secrétaire d'État Hillary Clinton a lancé le programme "Feed the Future ", une initiative qui s'inspire des principes du sommet de la FAO à Rome (novembre 2009) et vise deux objectifs : 1) investir dans la production agricole des pays en voie de développement en les impliquant directement dans le développement et la gestion de nouveaux projets ;2) améliorer l'accès des populations pauvres aux programmes de nutrition. (White House, 2010; Chicago Council on Global Affairs, 2010). Contrairement aux autres programmes d'aide alimentaire américains, principalement financés par le département de l'Agriculture, le financement de Feed the Future (1,8 milliards en 2011) s'appuie sur les crédits des départements d'État, du Trésor et de l'USAID. À ces dépenses s'ajoute la contribution américaine au financement du nouveau Programme mondial pour l'agriculture et la santé alimentaire au sein de la Banque mondiale qui s'élève à 408 millions de dollars. Au total, ces investissements 
représenteraient une hausse de $40 \%$ par rapport au budget de 2010 et pourraient compenser la baisse du montant de l'aide alimentaire allouée via le département de l'Agriculture (CRS, 2011). Néanmoins, l'avenir de ces nouveaux investissements est aujourd'hui incertain au vu des récentes réductions budgétaires imposées au département d'État et à l'USAID. Ainsi, la réorientation de l'aide alimentaire vers les investissements productifs agricoles reste prisonnière des conflits budgétaires entre le Congrès et l'Exécutif.

21 Par ailleurs, deux obstacles structurels empêchent toujours les États-Unis de lutter efficacement contre l'insécurité alimentaire mondiale: le soutien à la production de biocarburants; le conditionnement de l'aide alimentaire à l'utilisation de produits et services américains. Dans le premier cas, Barack Obama a perpétué la politique de son prédécesseur en apportant un soutien constant à la production d'éthanol, malgré les effets inflationnistes de cette politique sur les marchés alimentaires mondiaux. Cette question, comme celle du système de préférence nationale est régie par la loi agricole de 2008, qui définit aussi bien les modalités d'acheminement de l'aide alimentaire que les subventions aux producteurs d'éthanol. Toute réforme de cette loi impliquerait d'affronter une puissante coalition d'intérêts agricoles qui jouissent de solides appuis au Congrès - au sein des Démocrates comme des Républicains. Barack Obama a déjà mesuré les risques d'une telle confrontation lors de sa tentative de plafonner les subventions aux exploitations agricoles à 250000 dollars en 2009 - un conflit qui s'est soldé par un échec du président américain. Ainsi, en dépit de l'urgence déficitaire qui domine l'agenda politique américain en 2011, le système des subventions agricoles reste une question épineuse.

\section{Conclusion}

22 Pour conclure, la politique de l'aide extérieure américaine face à la crise alimentaire a évolué non pas tant au gré de la conjoncture économique mondiale qu'en fonction des priorités politiques des occupants de la Maison Blanche et du contexte politique washingtonien. L'administration Bush a adopté une politique paradoxale à deux égards. Si elle a répondu à l'urgence humanitaire en obtenant une augmentation des budgets de l'alimentaire, elle a aussi affaibli le processus même de l'aide extérieure en diminuant progressivement le montant des crédits annuels fixes. En outre, sa volonté tardive de rationaliser la politique d'aide extérieure américaine n'a pas suffi à compenser la fragmentation institutionnelle de la politique extérieure américaine liée à la multiplication des programmes d'aide extérieure sous la présidence de George W. Bush. Enfin, le président républicain n'est pas parvenu à réformer le système de préférence nationale qui continue à gripper l'aide alimentaire américaine.

23 L'administration Obama a elle aussi tenté de remédier à certains défauts structurels de l'APD en misant sur les investissements productifs agricoles dans les pays en voie de développement. Comme son prédécesseur, le président démocrate a cherché à améliorer la coordination des différents services de la politique étrangère américaine. Ses efforts se sont toutefois heurtés aux réticences du Congrès, qui constitue la pierre d'achoppement à toute réforme profonde de la politique de l'aide extérieure. Indépendamment de la politique menée par l'Exécutif, la capacité des États-Unis à répondre efficacement à une crise humanitaire reste tributaire de la volonté du Congrès à agir, non seulement en vertu de ses pouvoirs budgétaires mais de ses intérêts 
à préserver le status quo dans le domaine agricole. À moins que la crise alimentaire mondiale ne prenne une dimension humanitaire encore plus tragique, la politique de l'aide alimentaire américaine devrait souffrir encore longtemps de ses défaillances structurelles.

\section{BIBLIOGRAPHIE}

Anon., « Whatever happened to the food crisis ?», The Economist, 4 juillet 2008, p. 53-54.

Anon., « Corn Power ; Sen. Grassley Snuffs Out Dissent on Ethanol Subsidies », The Washington Post, 5 août 2009, p. 12.

Bilmes, Linda et Joseph Stiglitz « The Iraq War Will Cost Us \$3 Trillion, and Much More », The Washington Post, 9 mars 2008, p. 1.

Brainard, Lael, « U.S. Foreign Assistance : Reinventing Aid for the $21^{\text {st }}$ Century », House Subcommittee on State, and Related Programs, Washington, DC, 23 janvier 2008.

Bush, George W., « The National Security Strategy of the United States of America », Washington, DC, 2002.

Capehart, Tom, Randy Schnepf et, Brent D. Yacobucci, « Biofuels Provisions in the 2007 Energy Bill and the 2008 Farm Bill : A Side-by-Side Comparison », Congressional Research Service, Washington, DC, 2008.

Carlsson, Bjorn Tore, Carlos Buhigas Schubert et Sarah Robinson, « Aid Effectiveness Agenda : Benefits of a European Approach », Bruxelles, Commission Européenne, 2009.

Chicago Council on Global Affairs, « Details on the Obama Administration's FY2011 Budget Request to Congress », 2010. Disponible en ligne :

http://www.thechicagocouncil.org/UserFiles/File/GlobalAgDevelopment/Newsletter/ CCGAFY2011BudgetAnalysis.pdf. Page consultée en septembre 2011.

Clinton, Hillary R., «Statement of Senator Hillary Rodham Clinton Nominee for Secretary of State ", Senate Foreign Relations Committee, Washington, DC, 13 janvier 2009. Disponible en ligne : http://www.state.gov/secretary/rm/2009a/01/115196.htm. Page consultée en septembre 2011.

Conférence des Nations Unies sur le commerce et le développement, « Répondre à la crise alimentaire globale », New York / Genève, Nations Unies, 2008.

Epstein, Susan B., «Foreign Aid Reform : Issues for Congress and Policy Options », Congressional Research Service, Washington, DC, 2009.

Food and Agriculture Organization of the United Nations, « Food Price Indices », 2009, http:// www.fao.org/worldfoodsituation/en/. Page consultée en août 2011.

Food and Agriculture Organization of the United Nations, « L'état de l'insécurité alimentaire dans le monde. Crises économiques - répercussions et enseignements », 2009. Disponible en ligne : ftp://ftp.fao.org/docrep/fao/012/i0876f/i0876f.pdf. Page consultée en octobre 2009. 
Hanrahan, Charles E., « Rising Food Prices and Global Food Needs : The U.S. Response », Congressional Research Service, Washington, DC, 2008. Disponible en ligne : http:// assets.opencrs.com/rpts/RL34478_20080508.pdf. Page consultée en octobre 2009.

Herrling, Sheila et Steve Radelet, « Modernizing U.S. Foreign Assistance for the Twenty-first Century ", in Nancy Birdsall, The White House and the Word. A Global Development Agenda for the Next U.S. President, Center for Global Development, Washington, DC, 2009, p. 273-298.

Ho, Melissa D. et Charles E. Hanrahan, « The Obama Administration's Feed the Future Initiative ", Congressional Research Service, Washington, DC, 2011. Disponible en ligne : http://www.fas.org/ sgp/crs/misc/R41612.pdf, Page consultée en septembre 2011.

International Monetary Fund, «Impact of High Food and Fuel Prices on Developing Countries », 2009. Disponible en ligne : http://www.imf.org/external/np/exr/faq/ffpfaqs.htm. Page consultée en octobre 2008

Kharas, Homi, « The Reality of Rising Food Prices : Benefits to the Poor », The Brookings Institution, 2008. Disponible en ligne :

http://www.brookings.edu/opinions/2008/0806_food_prices_kharas.aspx. Page consultée en septembre 2011.

Lacombe, Clément, « Nourrir la planète en 2050, un défi déjà d'actualité », Le Monde, 17 octobre 2009, p. 4.

LaFranchi, Howard, « Despite Crisis, Bush Urges US Allies to Maintain Foreign Aid », Christian Science Monitor, 23 octobre 2008, p. 2.

Obama, Barack H., « Remarks of Senator Barack Obama to the Chicago

Council of Global Affairs », Chicago, 23 avril 2007. Disponible en ligne : http:// my.barackobama.com/page/content/fpccga/. Page consultée en octobre 2009.

Organisation for Economic Cooperation and Development, « Aid Statistics, Donor Aid Charts ", 2010. Disponible en ligne : http://www.oecd.org/dataoecd/42/30/44285539.gif. Page consultée en mars 2010.

Oxfam International, « Food Aid or Dumping ? Separating Wheat from Chaff », Oxfam Briefing Paper, 2005. Disponible en ligne : http://www.farmingsolutions.org/pdfdb/ oxfamreportUS.pdf. Page consultée en octobre 2009.

Rose, Frank, « Investment in United States Futures and Options Markets : A Discussion of the Possible Impact on Commodity Prices ", 2009. Disponible en ligne : http://www.fao.org/docrep/ 011/ai482e/ai482e12.htm, Page consultée en septembre 2011.

Sen, Amartya, « The Rich Get Hungrier », The New York Times, 28 mai 2008, p. 21.

U.S. Department of Agriculture (USDA), « Farm Bill Proposal », 2007. Disponible en ligne : http:// www.usda.gov/documents/07finalfbp.pdf. Page consultée en octobre 2009.

U.S. Department of State, « Congressional Budget Justification. Foreign Assistance. Summary Tables. Fiscal Year $2011 », 2010$. Disponible en ligne : http://www.state.gov/ documents/organization/138174.pdf. Page consultée en septembre 2011.

U.S. Department of State, « International Affairs - FY 2010 Budget », 2009. Disponible en ligne : http://www.state.gov/r/pa/prs/ps/2009/05/123160.htm. Page consultée en octobre 2009.

U.S. Department of State, «The Department of State's Quadrennial Diplomacy and Development Review », 2009. Disponible en ligne : http://www.state.gov/r/pa/prs/ps/2009/july/ 125956.htm . Page consultée en octobre 2009. 
U.S. Department of State, « Congressional Budget Justification. Foreign Operations. FY 2010 Budget Request », 2009 Disponible en ligne : http://www.state.gov/ documents/organization/123415.pdf. Page consultée en octobre 2009.

United States Government Accountability Office, « Foreign Assistance. Various Challenges Impede the Efficiency and Effectiveness of U.S. Food Aid », Washington, DC, 2007.

United States Government Accountability Office, « Foreign Aid Reform. Comprehensive Strategy, Interagency Coordination, and Operational Improvements Would Bolster Current Efforts », Washington, DC, 2009.

US Agency for International Development, US Department of Agriculture, « U.S. International Food Assistance Report 2008 », Washington, DC, 2008.

USAID, Bureau for Democracy, Conflict, and Humanitarian Assistance (DCHA). Office of U.S. Foreign Disaster Assistance (OFDA). « Global Food Insecurity and Price Increase », Washington, DC, 2009.

Velut, Jean-Baptiste, «La politique commerciale de George W. Bush : rupture ou continuité ? », La Revue LISA, vol. 8, n¹, 2010, p. 154-166.

Washington Post, « What's Getting Cut in the FY 2011 Budget?», 12 avril 2011. Disponible en ligne : http://www.washingtonpost.com/blogs/federal-eye/post/whats-getting-cut-in-thefy-2011-budget/2011/04/11/AFMIynLD_blog.html. Page consultée en août 2011.

White House, « President Obama's Global Development Policy and Global Food Security », September 22, 2010. Disponible en ligne :

http://www.whitehouse.gov/sites/default/files/Food_Security_Fact_Sheet.pdf. Page consultée en septembre 2011.

World Food Programme, « WFP Donors », 2009. Disponible en ligne : http://www.wfp.org/about/ donors/wfp-donors. Page consultée en octobre 2009.

\section{NOTES}

1. Calculs de l'auteur à partir des statistiques de la FAO, disponibles sur : http://www.fao.org/ worldfoodsituation/en/ .

2. Certains analystes ont dénoncé l'alarmisme des institutions internationales en soulignant les bénéfices à long terme de la hausse des prix des denrées alimentaires pour l'agriculture des pays en voie de développement. Voir Kharas (2008).

3. Il faut toutefois préciser que cette augmentation a été très inégale: l'augmentation de production céréalière a eu lieu principalement dans les pays riches (+11\% en 2008). Si l'on exclut la Chine, le Brésil et l'Inde, la production dans les pays en voie de développement a en réalité diminué (The Economist, 2008).

4. Selon le FMI, la moitié de l'augmentation de la consommation des principales récoltes alimentaires serait liée à la production d'agrocarburants et aux politiques incitatives mises en place par les États-Unis et l'Union européenne. Voir FMI (2009).

5. Voir section suivante.

6. Depuis 1970, la consommation moyenne de viande par habitant à l'échelle mondiale est passée de 25 à $38 \mathrm{~kg}$ par an. Il faut quatre calories végétales pour produire une seule calorie animale dans le cas du porc, et onze calories végétales pour le bœuf ou le mouton (Lacombe, 2009).

7. Pour plus de détails, lire Velut (2010). 
8. Les termes d'aide extérieure et d'aide publique au développement sont ici utilisés de manière interchangeable et reprennent la définition statistique du Comité de l'aide pour le développement (CAD) de l'Organisation pour la coopération et le développement économique (OCDE). Cet indicateur exclut certaines formes d'aide militaire et sécuritaire. Pour une brève discussion des différentes mesures de l'aide extérieure, lire CRS (2009 : 1-2).

9. Il s'agit ici d'une mise à jour du travail de Radelet (2009) à partir du rapport du CAD de 2009 (OCDE, 2010).

10. Créé en 2002, le Millennium Challenge Account vise à transformer le processus de l'aide au développement en conditionnant cette dernière à une série de critères politiques comme la gouvernance démocratique, l'investissement en matière d'éducation et de santé, le respect des principes du libre-marché, etc.

11. En 2006, la somme de l'aide accordée à l'Irak et à l'Afghanistan représentait plus de $25 \%$ de l'APD américaine (Radelet, 2009).

12. Au terme de la présidence Bush, le département de la Défense gère plus d'un cinquième du budget de l'aide extérieure américaine, contre seulement $6 \%$ en 2002 (ibid.).

13. Pour plus de détails, lire Brainard (2009).

14. Le montant de l'aide alimentaire se calcule sur la base des crédits budgétaires alloués à six programmes qui dépendent de l'Agence pour le développement international et des Départements d'État et de l'Agriculture. Pour plus de détails, voir GAO (2007, annexe 2).

15. Cette estimation s'appuie sur les données de Joseph Stiglitz et ses associés (Bilmes \& Stiglitz, 2008).

16. Une autre clause, abrogée par le Congrès en 1995, prévoyait que $25 \%$ de l'aide alimentaire transite par les ports des Grands Lacs (US GAO, 2007).

17. Selon un récent rapport de la Commission européenne, $15 \%$ de l'aide extérieure de l'Europe des 15 reste liée à des préférences nationales (Carlsson, Schubert \& Robinson, 2009).

18. Ces efforts de coordination ne concernent ni les autres acteurs de l'APD comme la Millennium Challenge Corporation, PEPSAR, ni une douzaine d'agences gouvernementales rattachés à l'exécutif (Radelet, 2009).

19. La loi sur l'énergie (Energy Independence and Security Act) de 2007 prévoit en effet d'augmenter l'utilisation des agrocarburants pour atteindre l'objectif de 9 milliards de gallons en 2008 et 36 milliards en 2022. Pour une comparaison avec les objectifs de la loi énergétique de 2005, lire Capehart, Schnepf, \& Yacobucci (2008).

20. Même en incluant la somme de 880 millions de dollars allouée à l'aide internationale aux catastrophes (international disaster assistance) - habituellement exclue des calculs de l'aide alimentaire - le budget total reste bien inférieur au montant peu crédible (et dépourvu d'explication) de 3,4 milliards affiché par le Département d'État pour 2010 (US DOS, 2009b).

\section{RÉSUMÉS}

Selon l'Organisation des Nations Unies pour l'alimentation et l'agriculture (FAO), l'augmentation des prix des denrées alimentaires en 2007-2008 aurait fait sombrer dans la faim et la pauvreté chroniques près de 100 millions de personnes, portant leur nombre total à un triste record de 1,02 milliards de personnes en 2009. Malgré l'ampleur de ce désastre humanitaire, «l'autre crise » et les initiatives politiques visant à l'enrayer ont reçu beaucoup moins d'attention que la 
crise financière internationale de 2008-2009. Cette communication se propose d'analyser les initiatives politiques adoptées par le gouvernement américain face à cette crise - de George W. Bush à Barack Obama sans oublier le rôle clé joué par le Congrès américain. L'étude de ces initiatives conjoncturelles s'accompagne d'une réflexion sur le processus décisionnel de l'aide extérieure américaine, tant du point de vue de ses objectifs que de ses modalités et de ses lacunes.

According to the United Nations Food and Agriculture Organization (FAO), the increase in food prices in 2007-2008 resulted in poverty and hunger for nearly one hundred million people, raising their number to a tragic record of 1,02 billion people in 2009. Despite the magnitude of this human disaster, the "other crisis" and the political responses aimed at stemming this phenomenon have drawn much less attention from economists and political scientists than the international financial crisis of 2008-2009. This contribution seeks to analyze the policy initiatives undertaken by the US government in response to the food crisis - from George W. Bush to Barack Obama including the key role played by the US Congress. The study of these short-term initiatives is followed by a deeper analysis of the decision-making process of US foreign aid focusing on its objectives, mechanisms and shortcomings.

Según la Organización de las Naciones Unidas para la Agricultura y la Alimentación (FAO), el aumento de los precios de alimentos en 2007-2008 tuvo como consecuencia la pobreza y el hambre para unos cien millones de personas, subiendo este número a un récord trágico de 1,02 mil millones en 2009. A pesar de la importancia del desastre, la « otra crisis » y las iniciativas políticas para detener este fenómeno han preocupado mucho menos a los economistas y politólogos que la crisis financiera internacional. Esta contribución se esfuerza por analizar las iniciativas políticas del gobierno estadounidense en respuesta a la crisis de alimentos - desde la administración de George W. Bush hasta Barack Obama - mencionando también el papel crucial del Congreso de los EE.UU. El estudio de estas iniciativas coyunturales se completa con el análisis del proceso de toma de decisión de la ayuda exterior estadounidense con un enfoque en sus objetivos, modalidades y lagunas.

\section{INDEX}

Mots-clés : aide extérieure, crise alimentaire, politique étrangère, pauvreté, États-Unis, George W. Bush, Barack Obama

Palabras claves : ayuda exterior, crisis de alimentos, política exterior, pobreza, Estados Unidos, George W. Bush, Barack Obama

Keywords : Foreign aid, food crisis, foreign policy, poverty, United States, George W. Bush, Barack Obama

\section{AUTEUR}

\section{JEAN-BAPTISTE VELUT}

Jean-Baptiste Velut est Maître de Conférences en Civilisation Américaine à l'Université Sorbonne Nouvelle - Paris III. jbvelutatgmailpointcom 